УДК 514.7

DOI: $10.21779 / 2542-0321-2019-34-4-65-71$

\title{
Т.И. Уткина
}

\section{Вопросы теории три-тканей и их приложения}

Орский гуманитарно-технологический институт (филиал) Оренбургского государственного университета; Россия, Оренбургская обл., 462403, г. Орск, пр. Мира, 15a; UtkinaTI@yandex.ru

Объектом исследования данной работы являются: три-ткани и их использование в обосновании проективной геометрии и в построении модели газа, в нахождении относительных инвариантов три-тканей; исследование параллелизуемости плоских и криволинейных три-тканей, заданных дифференциальными уравнениями; проектирование культурно-просветительских программ по популяризации теории три-тканей и их приложений в реальной жизни общества.

Цель работы - разработка различных направлений использования теории три-тканей в проективной геометрии, классификации дифференциальных уравнений, построении геометрических моделей физических закономерностей.

В результате исследования впервые разработана технология, ориентированная на формирование профессиональной компетентности будущего бакалавра педагогического образования в разработке и реализации культурно-просветительских программ в условиях изучения дисциплины «Вопросы теории три-тканей» из вариативной части основных образовательных программ по направлению подготовки Педагогическое образование (с одним и двумя профилями подготовки «Математика» и «Математика, физика»). Технология обоснована методологически, фактологически, аргументирована с позиции результатов педагогического эксперимента и анализа фактического участия студентов в научно-исследовательской работе по вопросам теории три-тканей. Рассмотрены приложения теории три-тканей в аксиоматическом обосновании проективной геометрии плоскости, в построении геометрических моделей физических закономерностей и в профессиональной подготовке учителя математики к организации работ по популяризации математических знаний через привлечение студентов к научно-исследовательской работе по некоторым вопросам теории три-тканей, связанным с построением изображений шестиугольных три-тканей. Проведенное исследование «Три-ткани и их приложения» прошло государственную регистрацию во Всероссийском научно-техническом информационном центре (регистрационный номер 01201352349).

Ключевые слова: три-ткань, модель, профессиональная подготовка учителя математики.

В статье рассматриваются вопросы математического моделирования для аксиоматического построения проективной геометрии плоскости, создания геометрической модели состояния газа и конструирования педагогической технологии профессиональной подготовки будущих учителей математики к организации работ по математическому просвещению и популяризации математической науки на основе теории три-тканей.

В настоящей работе применяются следующие термины с соответствующими определениями.

Инцидентность - отношение принадлежности, связывающее точки и прямые.

Трехвершинник - совокупность трех точек, не принадлежащих одной прямой, и трех прямых, проходящих через эти точки.

Серия 1. Естественные науки. 2019. Том 34. Вып. 4 
Дезаргово предложение - если для двух трехвершинников существует биективное соответствие между их вершинами такое, что прямые, инцидентные соответствующим вершинам трехвершинников, инцидентны одной точке $S$, то точки, инцидентные соответствующим сторонам, инцидентны одной прямой $l$. Точку $S$ называют дезарговой точкой, а прямую $l$ - дезарговой прямой.

Пучок прямых линий - множество прямых плоскости, инцидентных одной точке, называемой вершиной пучка.

Три-ткань множества точек $\Omega$ и три семейства линий $\left\{L_{1}, L_{2}, L_{3}\right\}$ области $\Omega$ называют плоской три-тканью, если через каждую точку области $\Omega$ проходит только одна линия из каждого семейства, линии из одного семейства не пересекаются, а линии из разных семейств не касаются и пересекаются не более чем в одной точке.

Условные обозначения: $P i j$ - линия под номером $j$ в пучке с вершиной в точке $P i$, $C=B_{2} \cap O_{3}-$ точка $C$ есть точка пересечения линии из пучка с вершиной в точке $B_{2}$ с линией из пучка $O_{3}$.

Понятие три-ткани позволяет естественным образом интегрировать различные математические структуры. Три-ткань - математическая структура, можно сказать, универсального уровня, позволяющая выявить подход, направленный на формирование целостного представления о математических структурах и их практических приложениях, в том числе и в работе по математическому просвещению и популяризации математики.

Три-ткань, рассматриваемая с точностью до локальных диффеоморфизмов, однозначно определяется своим уравнением $z=f(x, y)$, где $f$ - произвольная гладкая функция. Поэтому теория три-тканей имеет разнообразные приложения в разных разделах математики и физики. Но геометрия три-тканей тесно связана и со многими другими «классическими» областями математики. В первую очередь можно выделить: вопросы аксиоматического обоснования элементарной и проективной геометрии; алгебраическую теорию групп и теорию непрерывных групп Ли; проективную и алгебраическую геометрии; классическую дифференциальную геометрию Гаусса; проективную дифференциальную геометрию; риманову геометрию и ее обобщения; вариационное исчисление; теорию функций; формы Пфаффа и дифференциальные уравнения; теорию расслоенных пространств; теорию квазигрупп и луп; конформную геометрию.

Сущностные характеристики понятия три-ткани связаны с криволинейной тканью и методом внешних форм. Криволинейная ткань рассматривается как совокупность нескольких семейств из гладких кривых, заданных на плоскости, на поверхности однородного пространства или на некотором гладком двумерном многообразии. Через каждую точку области определения проходит по одной линии из каждого семейства, линии ткани в каждой точке трансверсальны, и у каждой точки есть окрестность, в которой каждое из семейств образует слоение.

Криволинейные ткани различают по количеству семейств (2-ткани или сети, триткани, $d$-ткани) и по отношению эквивалентности (локального диффеоморфизма, сохраняющего трансверсальность слоев).

Основной геометрический образ в дифференциально-топологической теории тканей - конфигурации, образованные слоениями ткани. При локальных диффеоморфизмах сохраняется свойство конфигураций быть замкнутыми.

Заметим, что в дифференциально-топологической теории тканей не имеет смысла рассматривать сети (2-ткани), поскольку подходящим локальным диффеоморфизмом всякую сеть можно отобразить (локально) на декартову сеть. Но три-ткани уже пред-

66 Вестник Дагестанского государственного университета

Серия 1. Естественные науки. 2019. Том 34. Вып. 4 
ставляют собой нетривиальный объект, т. к. не существует локального диффеоморфизма, который все три семейства отображает на три семейства параллельных прямых.

Поскольку два семейства линий произвольной три-ткани $W$ всегда можно «выпрямить» и принять за декартову сеть, то в некоторой окрестности ее (т. е. три-ткань) можно задать уравнением $z=f(x, y)$, причем линии третьего семейства суть линии уровня функции $f$. Ограничение на функцию $f(x, y)$ минимально: уравнение ткани должно быть однозначно разрешимо (локально) относительно каждой из переменных $x$ и y. Последнее обстоятельство делает теорию тканей приложимой к любой теории, где объектом изучения является гладкая функция двух переменных. Это, например, некоторые физические законы, двусторонне разрешимые бинарные операции (квазигруппы и лупы), дифференциальные уравнения и т. д. $[6,7]$.

Важным примером три-ткани служит ткань на плоскости, образованная тремя пучками прямых $P\left(S_{1}\right), P\left(S_{2}\right), P\left(S_{3}\right)$ с вершинами в точках $S_{i}$, при условии, что прямые $S_{1} S_{2}, S_{2} S_{3}, S_{3} S_{1}$ удалены.

Ткань называется параллельной, если она образована тремя семействами из параллельных прямых линий. Три-ткань, гомеоморфная параллельной, называется параллелизуемой. Три-ткань, состоящая из прямых линий, называется прямолинейной. Не всякая прямолинейная три-ткань является параллелизуемой.

Исключая переменные $x$ и $y$ из уравнений $F_{1}(x, y)=u_{1}, F_{2}(x, y)=u_{2}, F_{3}(x, y)=u_{3}$, получим $\Phi\left(u_{1}, u_{2}, u_{3}\right)=0$. Это уравнение называют уравнением ткани, а функцию $\Phi$ - функцией ткани.

Для параллелизуемой три-ткани $z=f(x, y)$, и только для нее выполняется условие Сен-Робера

$$
\frac{\partial z}{\partial x \partial y} \ln \left(\frac{f_{x}}{f_{y}}\right)=0 .
$$

С каждой плоской три-тканью связывают так называемую шестиугольную фигуру. Условимся линии три-ткани, проходящие через некоторую точку $M$ из области определения, обозначать $M_{i}$ - линия из $i$-того семейства (здесь индекс $i$ принимает значения $1,2,3)$. С учетом принятого обозначения алгоритм построения шестиугольной фигуры следующий. Через точку $A$ из области определения проводим линии $A_{1}, A_{2}, A_{3}$. На линии $A_{3}$ выбираем точку $B$ и строим точку $O$ пересечения линий $A_{2}$ и $B_{1}$. Далее строим последовательно точки

$C=B_{2} \cap O_{3}, F=A_{1} \cap O_{3}, D=C_{1} \cap A_{2}, E=D_{3} \cap B O, G=E_{2} \cap A_{1}$. Фигура $A B C D E G$ называется шестиугольной. Заметим, точки $A_{1}, F$ и $G$ коллинеарные. В том случае, когда точки $F$ и $G$ совпадают, говорят, что шестиугольная фигура $A B C D E F$ замыкается. Если на три-ткани замыкаются все шестиугольные фигуры, то такая ткань называется шестиугольной. Шестиугольная ткань параллелизуема. Верно и обратное утверждение.

Замкнутая шестиугольная фигура является конфигурацией типа $(6,3 ; 9,2)$, т. е. через каждую точку проходит ровно три прямых линии и на каждой линии лежит по две точки (точка $O$ к конфигурации не относится). Если ткань образована тремя пучками прямых линий с вершинами $S_{1}, S_{2}, S_{3}$, то вершины шестиугольной фигуры вместе с вершинами пучков порождают правильную конфигурацию.

Следует заметить, что три-ткани могут выступать инструментом классификации дифференциальных уравнений с точностью до изотопических преобразований, в чем и сказывается их универсальность $[1,2,7]$.

Рассмотрим вопрос об использовании параллелизуемых тканей для аксиоматического обоснования проективной геометрии плоскости. 
Структура проективной плоскости может быть определена следующим образом. Она включает в себя множество $T$ точек, множество $P$ прямых, отношение инцидентности $I$ точек и прямых. Отношение инцидентности должно удовлетворять аксиомам.

1. Всякой прямой инцидентны по крайней мере две различные точки.

2. Всяким двум различным точкам инцидентна единственная прямая.

3. Всяким двум различным прямым инцидентна единственная точка.

4. Выполняется Дезаргово предложение и двойственное ему.

Аксиоме 4 соответствует правильная конфигурация $(3,10)$, состоящая из десяти точек и десяти прямых. Эта конфигурация обладает свойством - каждой точке инцидентны три прямые, а каждой прямой инцидентны три точки. Таким же свойством обладают шестиугольные фигуры в параллелизуемых плоских три-тканях. Отсюда возникает гипотеза - аксиоме 4 равносильно следующее предложение.

5. Всякая три-ткань, образованная тремя пучками прямых линий, шестиугольная.

Докажем равносильность аксиом 4 и 5, т. е. из аксиом 1, 2, 3, 4 следует утверждение 5 и обратно, из аксиом 1, 2, 3, 5 вытекает предложение 4.

Пусть имеет место утверждение 4. Покажем, что ткань, образованная тремя пучками прямых линий, шестиугольная.

Рассмотрим пучки с вершинами $P_{1}, P_{2}, P_{3}$. Линии этих пучков будем обозначать $P_{i j}\left(j\right.$-я линия, $i$-го пучка). На линии $P_{31}$ возьмем две произвольные точки $A$ и $B$. Построим последовательно точки $O=A P_{2} \cap B P_{1}, F=A P_{1} \cap O P_{3}, C=B P_{2} \cap O P_{3}, D=A P_{2} \cap C P_{1}$, $E=B P_{1} \cap D P_{3} . G=E A \cap B D$. Далее рассмотрим два трехвершинника $A B P_{2}$ и $E D P_{1}$. Зададим соответствие между их вершинами $A \rightarrow E, B \rightarrow D, P_{2} \rightarrow P_{1}$, как на рисунке 1 .

Соответственные стороны этих трехвершинников пересекаются в точках $P_{3}=A B \cap E D, O=B P_{2} \cap D P_{1}, C=A P_{2} \cap E P_{1}$. По построению точки $P_{3}, O$ и $C$ лежат на одной прямой. На основании аксиомы 4 (двойственное утверждение) точка $G$ служит дезарговой точкой и, следовательно, она принадлежит прямой $P_{1} P_{2}$. Рассмотрим трехвершинники $A F E$ и $B C D$.Установим соответствие $A \rightarrow D, F \rightarrow C, E \rightarrow B$. В этих трехвершинниках прямые, проходящие через соответственные вершины, пересекаются в точке $O$ (дезарговой точке).

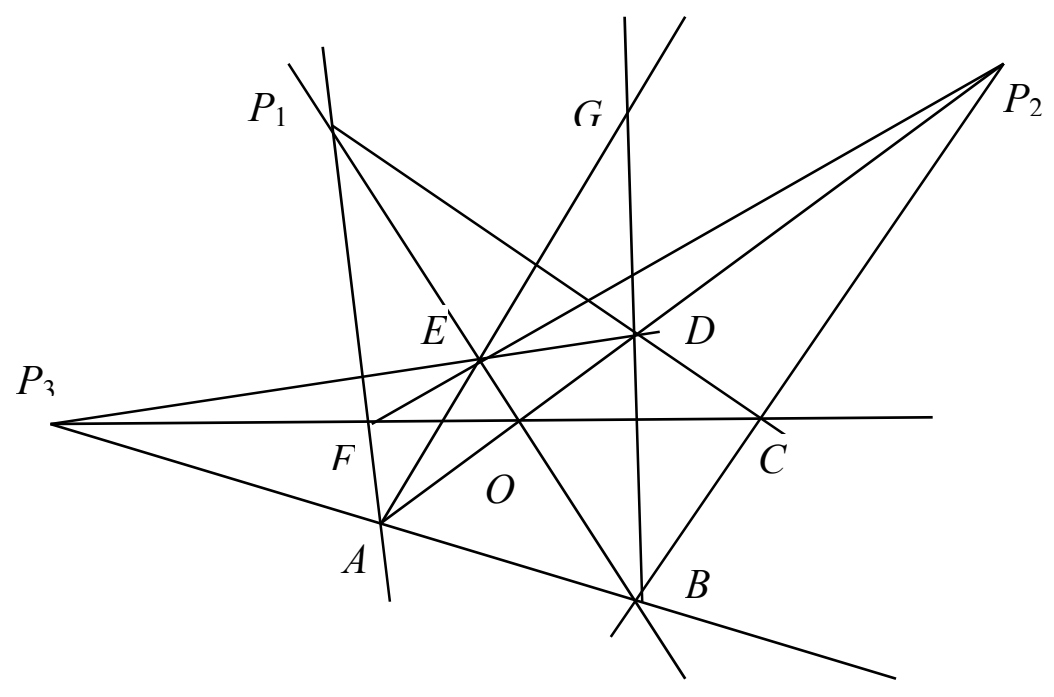

Рис. 1

Прямая $P_{1} G$ служит дезарговой прямой и сторона $B C$ пересекает ее в точке $P_{2}$. Следовательно, точки $F, E, P_{2}$ - коллинеарные точки, прямая $F E$ принадлежит пучку $P_{2}$. 
Таким образом, шестиугольная фигура $A B C D E F$ замкнутая, и ткань, образованная пучками $P_{1}, P_{2}, P_{3}$, шестиугольная [4].

Пусть теперь имеет место аксиома 5. Докажем, что дезаргово предложение будет выполняться. Через точку $O$ проведем три прямые и на каждой из них возьмем по две точки: $A$ и $D$ на первой прямой, $B$ и $E$ на второй, $C$ и $F$ на третьей, так чтобы никакие три точки не лежали на одной прямой. Эти шесть точек образуют два трехвершинника $A E F$ и $D B C$. Построим дополнительные точки $P_{1}=A F \cap D C, P_{2}=F E \cap B C, G=A E \cap D B$, $P_{3}=A B \cap C F, H=E F \cap C D, K=A F \cap B C$ в соответствии с рисунком 2.

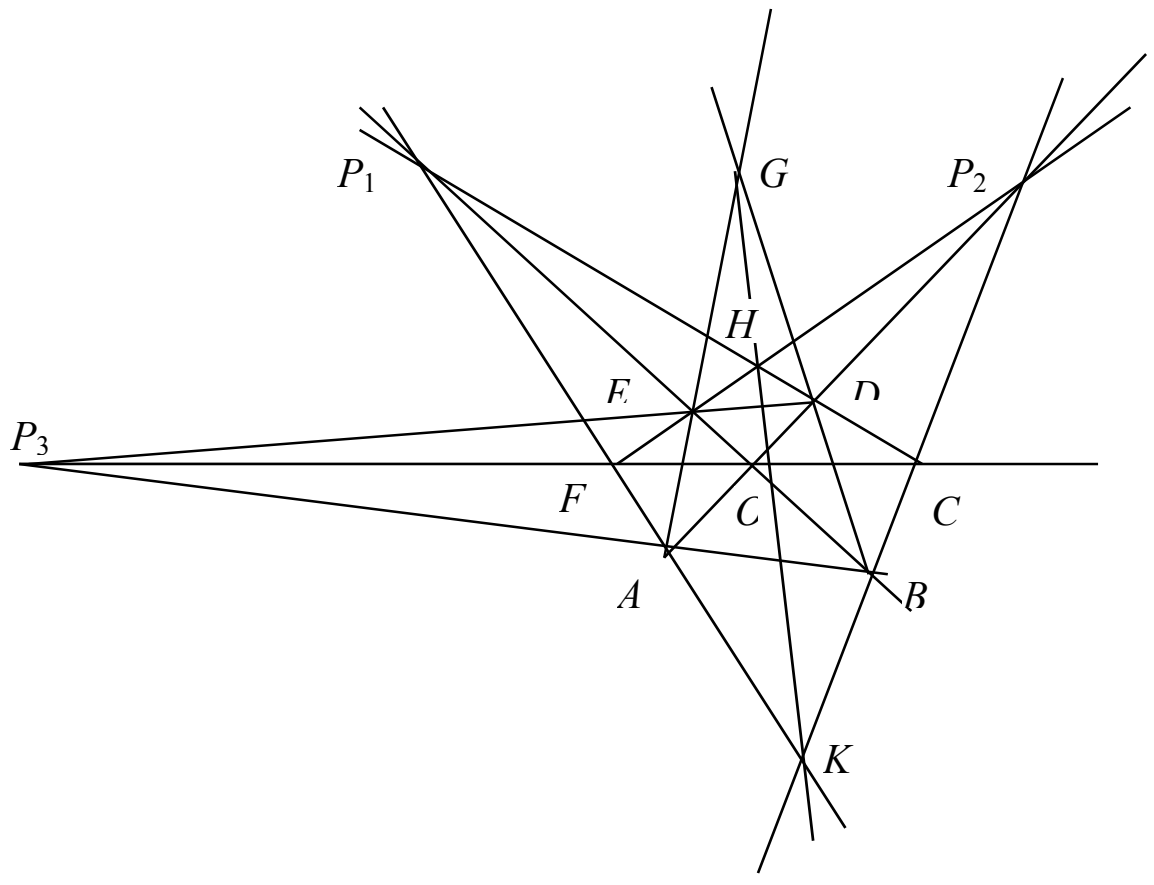

Рис. 2

Докажем, что точки $P_{3}, E, D$ лежат на одной прямой. Рассмотрим три-ткань, образованную пучками с вершинами $P_{1}, P_{2}, P_{1}$. Для этой три-ткани фигура $F A B C D E^{\prime}$, где $E^{\prime}=B P_{1} \cap D P_{3}$, является шестиугольной, и по аксиоме 5 она должна замыкаться, т. е. точка $E^{\prime}$ принадлежит прямым $B P_{1}$ и $F P_{2}$. Отсюда следует, что точки $E$ и $E^{\prime}$ совпадают, т. е. $E \in P_{3} D$. Зададим между вершинами трехвершинников $A E F$ и $B D C$ соответствие $A \rightarrow B, E \rightarrow D, F \rightarrow C$. Прямые линии $A B, F C$ и $E D$, проходящие через соответственные вершины, пересекаются в точке $P_{3}$. Соответственные стороны трехвершинников пересекаются в точках $G, H$ и $K$. Покажем, что эти точки коллинеарные. Рассмотрим триткань, образованную пучками с вершинами $P_{1}, G, P_{2}$. Для нее фигура $A K B D H E$ является замкнутой шестиугольной и, следовательно, точки $G, H$ и $K$ лежат на одной прямой. Таким образом, для дезарговых трехвершинников $A E F$ и $B D C$ точка $P_{3}$ - дезаргова точка, прямая $G K$ - дезаргова прямая [6].

Отсюда следует, что проективную геометрию плоскости можно строить на основе параллелизуемых три-тканей. Это дает возможность рассматривать в проективной теории плоскости задачи, связанные с шестиугольными фигурами.

Геометрическая (математическая) модель состояния газа в теории три-тканей представляется линиями, изображающими изопроцессы, которые образуют на гиперболическом параболоиде криволинейную три-ткань. Уравнение Менделеева-Клапейрона 
есть уравнение этой три-ткани, которая является шестиугольной, так как для нее выполняется условие Сен-Робера [3].

Результаты исследования положены в основу разработки модели подготовки будущих педагогов математики к организации работ по её популяризации. Разработанная модель ориентирована на формирование профессиональных компетенций будущего бакалавра педагогического образования в разработке и реализации культурнопросветительских программ в условиях изучения дисциплины «Вопросы теории тритканей» из вариативной части образовательных программ по направлению подготовки «Педагогическое образование» (с одним и двумя профилями подготовки «Математика» и «Математика, физика»). Суть модели состоит в овладении студентами методологическими знаниями по проектированию, планированию и организации работ по математическому просвещению и популяризации математики. Модель создана на основе компетентностного, процессного, интегративного, деятельностного и личностного подходов. В основу положены принципы важности математического образования для будущего страны, связи математики с жизнью и производственной практикой, преемственности и систематичности, гордости за достижения российских ученых, сочетания управления с развитием самостоятельности, согласованности требований ФГОС общего и высшего образования [9]. Структура модели состоит из следующих компонентов: ознакомление студентов с теоретическими основами популяризации математики; формирование компетенций по планированию и проектированию научно-популярных лекций; публичное предъявление научно-популярных лекций.

Экспериментально проверено положительное влияние разработанной модели на подготовку будущих педагогов-математиков к организации работ по математическому просвещению и популяризации математики $[5,8]$.

\section{Литература}

1. Уткин А.А., Шелехов А.М. Три-ткани, определяемые линейным дифференциальным уравнением // Изв. вузов. Математика. - 2001. - № 11. - С. 54-57.

2. Уткин А.А., Шелехов А.М. Три-ткани, определяемые уравнением Риккати // Изв. вузов. Математика. - 2004. - № 11. - С. 87-90.

3. Уткин А.А. Геометрическое моделирование окружающего мира. - Орск: Издательство Орского гуманитарно-технологического института (филиала) ОГУ, 2013. $215 \mathrm{c}$.

4. Салдайкина Н.В. Параллелизуемость плоской три-ткани, образованной тремя пучками прямых: материалы XVII Внутривузовской научно-практической конференции Орского гуманитарно-технологического института (филиала) ОГУ, посвященной 70-летию Победы в Великой Отечественной войне. - Ч. 3. Технические, физикоматематические и экономические науки. - Орск: Издательство Орского гуманитарнотехнологического института, 2015. - С. 112-115.

5. Уткина Т.И., Уткин А.А. Вопросы теории три-тканей в подготовке бакалавров к популяризации математики // Н.И. Лобачевский и математическое образование в России: материалы Международного форума по математическому образованию. - Казань: Изд-во Казан. ун-та, 2017. - Т. 2. - С. 166-170.

6. Три-ткани и их приложения: отчет о НИР (заключительный) / рук. НИОКТР Т.И. Уткина, исполн. А.А. Уткин Номер государственного учета отчета АААА-Б18218021490108-4 от 14.02.2018. - М., 2018.- 30 c.

7. Шелехов А.М., Лазарева В.Б., Уткин А.А. Криволинейные три-ткани: монография. - Тверь: Твер. гос. ун-т, 2013. -232 с. 
8. Стандартизация математического образования: проблемы внедрения и оценка эффективности: материалы XXXV Международного научного семинара преподавателей математики и информатики университетов и педагогических вузов. - Ульяновск: УлГПУ, 2016. - С. 180-183.

9. Концепция развития математического образования в Российской Федерации / Утверждена распоряжением Правительства Российской Федерации от 24 декабря 2013 г. № 2506-p.

Поступила в редакиию 4 августа 2019 г.

UDC 514.7

DOI: $10.21779 / 2542-0321-2019-34-4-65-71$

\section{Questions of the theory of three-webs and their applications}

\section{T.I. Utkina}

Orsk humanitarian technological institute (branch) of Orenburg state university; Russia, 462403, Orsk, Orenburg Region, Mir Avenue, 15a; UtkinaTI@yandex.ru

The objects of the work are: three-webs and their use in the justification of projective geometry and in the construction of a gas model, in finding the relative invariants of tri-tissues; the study of parallelizability of flat and curved three-webs given by differential equations, the design of cultural and educational programs on the popularization of the theory of three-webs and their applications in real life society.

The aim of the work is to develop different directions of using the theory of three-webs in projective geometry, classification of differential equations, construction of geometric models of physical laws.

As a result of the study the technology focused on the formation of professional competence of the future Bachelor of Pedagogy was first developed in the ability to develop and implement cultural and educational programs in the study of the discipline «Questions of the theory of three-webs» of the variable part of the main educational programs in the direction of Pedagogical education (with one and two profiles of training «Mathematics» and «Mathematics and Physics»). Technology is justified methodologically and factually, argued from the perspective of the results of the pedagogical experiment and the analysis of actual participation of students in research work on the theory of three-webs. Applications of the theory of three-webs in axiomatic justification of projective plane geometry, in construction of geometrical models of physical laws are considered as well as in professional training of a mathematics teacher for the popularization of mathematical knowledge through involvement of students in research work on some questions of the theory of three-webs connected with construction of images of hexagonal three-webs. The study "Three-webs and their applications" passed the state registration in the all-Russian scientific and technical information center (registration number 01201352349).

Keywords: three-web, a model, professional training of a mathematics teacher.

Received 4 August, 2019 